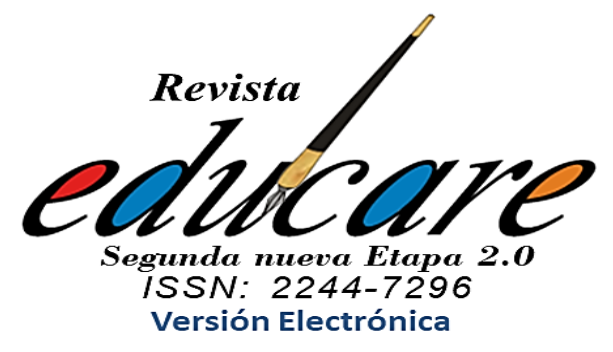

Volumen 25 No. 2 Mayo - Agosto 2021

(368-382)

\section{Ernesto Antonio De Lima Valdes \\ ORCID: https://orcid.org/0000-0001-6208-8640}

Ministerio de Educación (Colombia)
Doctorante en Educación Matemática, (UPEL-Maracay, Venezuela). Magíster Scíentíarum área: física de la universidad del Zulia (Venezuela), Especialista en Física General de la universidad del Atlántico, Licenciado en Matemáticas y Física de la universidad del Atlántico. Docente de aula titular de la secretaria de educación de Soledad (Atlántico) en el área de matemáticas y física de la institución educativa Francisco José de Caldas. Email: ernestomatematica@hotmail.com
Recibido:

29-03-2021

Aceptado:

22-06-2021

PROCESSING ELECTRICAL SHOCK IMAGES:

TOWARDS MATHEMATICAL AND INTERDISCIPLINARITY MODELING

PROCESANDO IMÁGENES DE DESCARGA ELÉCTRICA: HACIA LA MODELACIÓN MATEMÁTICA E INTERDISCIPLINARIDAD 

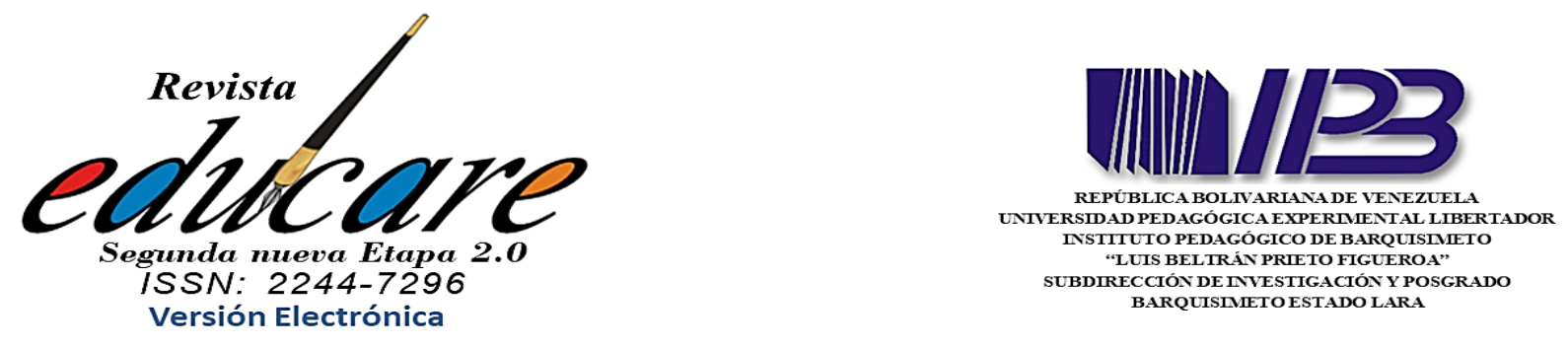

\title{
PROCESANDO IMÁGENES DE DESCARGA ELECTRICA: HACIA LA MODELACIÓN MATEMÁTICA E INTERDISCIPLINARIDAD
}

\author{
PROCESSING ELECTRICAL \\ SHOCK IMAGES: TOWARDS \\ MATHEMATICAL AND \\ INTERDISCIPLINARITY \\ MODELING
}

\section{Resumen}

Este artículo aborda la Transformada de Fourier y su aplicación en la modelación como conceptos relevantes para la matemática y las ciencias aplicadas. Para ello, se abordan diversos teóricos (Serrano; 2005, Alro y Skovsmose; 2004, Beyer; 1994 y Pimm; 1999) sobre el tema que aquí se desarrolla. Partiendo de la ejemplaridad y la interdisciplinariedad, se muestra el procesado digital de imágenes de descarga eléctrica y la transformada de Fourier. Como conclusiones se tienen que la Inversa de la Transformada de Fourier para el filtrado o eliminación de ruido de la imagen y la correlación digital se realiza aplicando la transformada de Fourier. La modelación matemática es un proceso fundamental en la actividad de aprendizaje científico-tecnológico para abordar problemas del contexto real práctico y además transformar cualitativamente los procesos de enseñanza en el ámbito de las ciencias aplicadas. Se hace énfasis en el hecho didáctico de que la Transformada de Fourier no es una teoría aislada sino de vital importancia y aplicabilidad en el campo científico y tecnológico.

Palabras clave: Procesado digital de imágenes, modelación matemática, interdisciplinariedad.

\section{Abstract}

This article addresses the Fourier Transform and its application in modeling as relevant mathematics and applied sciences concepts. For this, various theorists (Serrano; 2005, Alro and Skovsmose; 2004, Beyer; 1994 and Pimm; 1999) are approached on the subject that is developed here. Starting from the exemplary and interdisciplinarity, the digital processing of images of electric discharge and the Fourier transform are shown. The conclusions are that the Inverse Fourier Transform for filtering or elimination of image noise and digital correlation is performed by applying the Fourier transform. Mathematical modeling is a fundamental process in the activity of scientific-technological learning to address problems of the real practical context and also qualitatively transform the teaching processes in the field of applied sciences. Emphasis is placed on the didactic fact that the Fourier Transform is not an isolated theory but of vital importance and applicability in the scientific and technological field.

Keywords: Digital image processing, mathematical modeling, interdisciplinarity. 


\section{A Modo de Inicio}

\section{La Modelación Matemática En Un Contexto}

En las instituciones educativas la competencia matemática, por parte de los estudiantes, comprende los siguientes aspectos: el estudio y la comprensión de conceptos y algoritmos, la aplicación del lenguaje matemático, la solución de problemas y la demostración de propiedades. También abarca la discusión y comunicación de ideas matemáticas con su par, con el profesor o con otros miembros de la comunidad científica en general. Incluye, de igual modo, el desarrollo del pensamiento matemático y la habilidad para interpretar o diseñar modelos matemáticos referidos a las diversas situaciones de las ciencias aplicadas. La discusión y comunicación de ideas, dudas y errores en el contexto de una situación real juega un papel importante en la educación científica (Alro \& Skovsmose, 2004; Beyer, 1994; Pimm, 1999; Serrano, 2005).

La competencia matemática involucra un aspecto que ha sido central en el desarrollo de la misma matemática: la resolución y planteamiento de problemas. Estas actividades están presentes, aunque guiadas por una diversidad de enfoques, en el proceso de enseñanza y aprendizaje en nuestra educación matemática y ciencias aplicadas, y tienen un peso especial en el diseño curricular de matemáticas en el nivel universitario colombiano. Pruebas como la del TIMSS, del PISA o del SINEA se estructuraron con base en la resolución de problemas; situación que se presenta además en las pruebas de ingreso a la Universidad. Al considerar la resolución de problema como algo difícil se generaliza cuando se considera que un estudiante es bueno o es malo para la matemática. Existe un criterio bastante generalizado que consiste en considerar la resolución de problemas catalogados como difíciles, como un indicativo de que se es "bueno" o que se comprende la matemática.

Se quiere destacar las siguientes apreciaciones: (1) de qué forma se está trabajando en el aula, porque al parecer no está contribuyendo a desarrollar las competencias que se plantean el currículo para el estudiante (reportes que se citaron anterior mente); y, (2) el tipo de problema que se estudia y se propone a los estudiantes, por parte del profesor y en los libros de texto.

En cuanto al segundo punto, el término problema ha tenido diferentes interpretaciones tanto en matemática como en educación matemática, incluso los profesores y estudiantes tienen 
diferentes perspectivas de lo que es un problema. Por lo tanto, es importante que, en la instrucción universitaria, la enseñanza de la matemática esté dirigida a mostrarse en toda su amplitud de tal manera que lenguaje, herramienta y formación se compaginen hacia una aplicación evidente en la realidad y se le encuentre sentido a la serie de teorías o enunciados que la conforman. El matemático debe entender que enseñar matemática no es igual a crear matemática, la diferencia está, entre otras, en darle vida y sentido a lo que enseña a través de la aplicación tanto en el campo interno como en diferentes disciplinas científicas, como, por ejemplo, la física. Hasta ahora lo que se ha producido es una transferencia poco útil, descontextualizada produciendo en el estudiante una actitud de rechazo hacia la matemática.

Es por ello que el estudio de la Transformada de Fourier y su inversa, no se puede realizar descontextualizada sino buscando un sentido y aplicabilidad en algunas disciplinas técnicas y científicas. Debe verse como una herramienta que ayuda a entender qué sucede en un campo específico, como por ejemplo las telecomunicaciones o su aplicación en el procesamiento de imágenes de descarga eléctrica y su modelación interdisciplinar. Caso específico de estudio en este ensayo.

\section{Desarrollo}

\section{Procesado de imágenes de descarga eléctrica: ejemplo de modelación e interdisciplinaridad}

El procesamiento de imagen utiliza especialmente técnicas de filtrado para eliminar el ruido y la segmentación o división de una imagen en sus partes constituyentes.

La teoría de los filtros de los sistemas lineales está fundamentada en la noción de respuesta impulsional $h(\xi, \eta)$ y su función de transferencia $H(u, v)$. En este caso, la teoría permite establecer que si se le aplica la respuesta impulsional a una señal de entrada $f(\xi, \eta)$ con espectro $F(u, v)$ se obtendrá una señal de salida $g(\xi, \eta)$ con espectro $G(u, v)$ teniendo en cuenta que la salida de un sistema lineal (LSI) puede ser calculada directamente en el dominio de la entrada convolucionando la entrada con la respuesta impulsional del sistema (Goodman (1968).

$$
g(\xi, \eta)=h(\xi, \eta) * f(\xi, \eta)
$$


Entonces por el teorema de convolución se cumple la siguiente relación en el dominio de la frecuencia:

$$
G(u, v)=H(u, v) F(u, v)
$$

Donde $G, H$ y $F$ son respectivamente las transformadas de Fourier de $g, h$ y $f$ (Mejía, 2004). Por tanto, la función de transferencia puede ser escrita como:

$$
H(u, v)=A_{H}(u, v) e^{-i \phi_{H}(u, v)}
$$

Donde a los valores $A_{H}(u, v)$ y $\phi_{H}(u, v)$ se les conoce como función de transferencia de amplitud del sistema y la segunda función de transferencia de fase del sistema. Si el sistema considerado es tal que tiene a $e^{-i \phi_{H}(u, v)}=1$ se tiene un filtro de amplitud; porque únicamente la función de transferencia en amplitud actúa sobre la entrada; mientras que si $A_{H}(u, v)=1$ se tiene un filtro de solo fase (Mereles, 2012).

La señal de salida del sistema se escribe así:

$$
g(\xi, \eta)=\mathfrak{I}^{-1}\{G(u, v)\}
$$

Donde $\mathfrak{J}^{-1}$ es la transformada de Fourier inversa, esta relación permite determinar los efectos de la función de transferencia de amplitud sobre el espectro de amplitud de entrada y los efectos de la función de transferencia de la fase sobre el espectro de fase de entrada, ver figura 4.

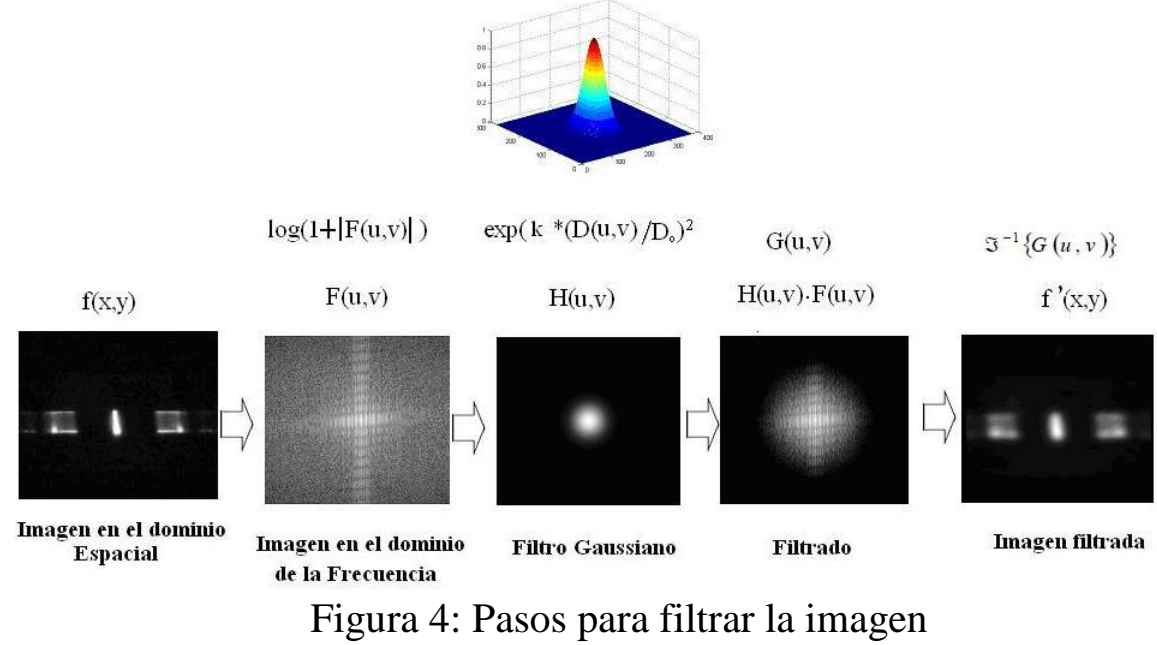


La segmentación consiste en separar en una imagen elementos que correspondan a unidades significativas. Ésta se realiza con base en criterios de "homogeneidad" como lo son tono, textura, profundidad, bordes, entre otros; aunque para tal fin también es posible considerar discontinuidades, movimiento (Ohta et al., 1980).

Para la segmentación de la imagen digital (espectro) se implementó la transformación del sistema de color RGB al sistema $I_{1} I_{2} I_{3}$ propuesto por Ohta et al. (1980). La forma matricial del sistema es:

$$
\left[\begin{array}{l}
I_{1} \\
I_{2} \\
I_{3}
\end{array}\right]=\left[\begin{array}{ccc}
\frac{1}{3} & \frac{1}{3} & \frac{1}{3} \\
\frac{1}{2} & 0 & -\frac{1}{2} \\
-\frac{1}{4} & \frac{1}{2} & -\frac{1}{4}
\end{array}\right] *\left[\begin{array}{l}
R \\
G \\
B
\end{array}\right]
$$

Utilizándose para segmentar las imágenes digitales la componente intensidad $I_{1}$ (Ohta et al., 1980), ver figura 5 .

$$
I_{1}=\frac{(R+G+B)}{3}
$$

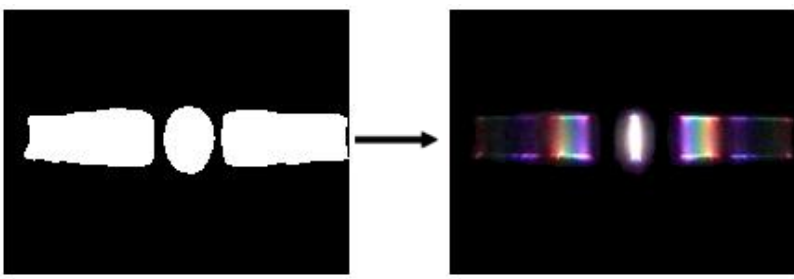

Figura 5: Segmentación del espectro

\section{Correlación Digital}

La correlación es un proceso que se utiliza en tratamiento de señales e imágenes, para comparar dos imágenes, este proceso puede ser realizado de forma óptica o de forma digital, en el caso del proceso óptico se requiere de una serie de lentes especiales para detectar las señales o imágenes a encontrar, mientras que en el procesamiento digital se requiere de un equipo de computación especializado (mayor velocidad) que pueda realizar este proceso. 
La correlación es el parámetro tradicionalmente utilizado para la detección y comparación de objetos, ya que, bajo ciertas restricciones, el valor de la correlación en el origen de un objeto consigo mismo (autocorrelación) es mayor que el de la correlación con cualquier otro objeto (Gonzalez \& Wintz, 1997).

Si $f(x, y)$ y $g(x, y)$ son funciones de variables continuas o discretas, su correlación cruzada $c(\alpha, \beta)$ se define así:

Para el caso continuo

$$
c(\alpha, \beta)=f(x, y) \circ g(x, y)=\iint_{-\infty}^{+\infty} f^{*}(\alpha, \beta) g(x+\alpha, y+\beta) d \alpha d \beta
$$

Para funciones discretas:

$$
c(x, y)=f_{e}(x, y) \circ g_{e}(x, y)=\sum_{m=0}^{M-1} \sum_{n=0}^{N-1} f_{e}^{*}(m, n) g_{e}(x+m, y+n)
$$

Donde * es el complejo conjugado

Para $\mathrm{x}=0,1,2, . ., \mathrm{M}-1$ e $\mathrm{y}=0,1,2, \ldots, \mathrm{N}-1 . f_{e}(x, y)$ y $g_{e}(x, y)$ son funciones extendidas, $\mathrm{y} \mathrm{M}, \mathrm{N}$ se escogen tal que eviten la superposición en los periodos de la función de correlación (Fernández et al., 2003).

Por medio de la transformada de Fourier es posible correlacionar dos imágenes o funciones $(f(x, y)$ y $g(x, y))$ de la siguiente manera:

Las Transformadas de Fourier $(F(u, v)$ y $G(u, v))$ de las imágenes se multiplican entre sí después de conjugar $\left(G^{*}(u, v)\right)$ una de ellas. La transformada de Fourier inversa de este producto $\left(\mathfrak{J}^{-1}\left\{f(u, v) G^{*}(u, v)\right\}\right)$ es la correlación entre las funciones $(c(\alpha, \beta)=f(x, y) \circ g(x, y))$. Se fundamenta en una de las propiedades de la Transformada de Fourier que garantiza su continuidad (Gualdrón, 2000). 


$$
\begin{aligned}
& f(x, y) \rightarrow F(u, v) \\
& \left.g(x, y) \rightarrow G(u, v) \rightarrow G^{*}(u, v)\right\} F(u, v) \cdot G^{*}(u, v) \rightarrow \mathfrak{J}^{-1}\left\{F(u, v) \cdot G^{*}(u, v)\right\}=f(x, y) \circ g(x, y)=c(\alpha, \beta)
\end{aligned}
$$

Cuando las funciones $f(x, y)$ y $g(x, y)$ son iguales la función $c(x, y)$ se le denomina autocorrelación. El módulo de la autocorrelación es máximo en el origen, es decir para desplazamiento cero ver figura 6.

$$
c(x, y)=\int_{-\infty}^{\infty} \int_{-\infty}^{\infty}|f(\alpha, \beta)|^{2} d \alpha d \beta
$$

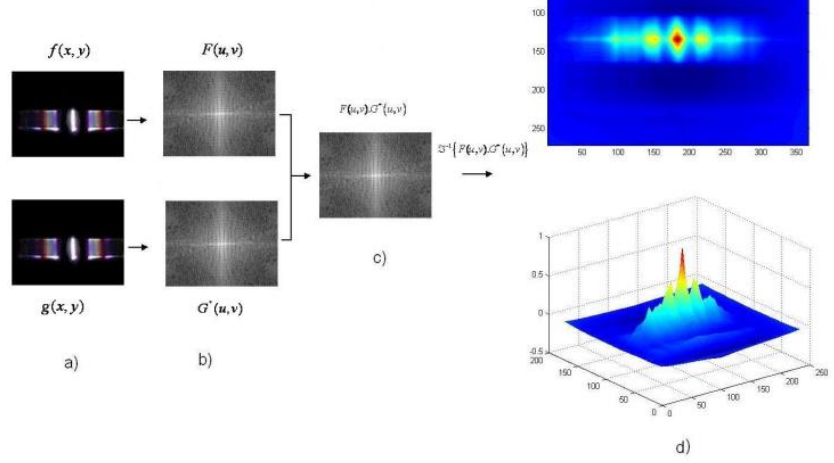

Figura 6. a) Espectro de una descarga eléctrica b) La FFT y el conjugado c) La multiplicación de FFT y su conjugado d) pico de la auto-correlación.

La correlación cruzada en el origen con otra función diferente es menor que la autocorrelación.

$$
\left|\int_{-\infty}^{\infty} \int_{-\infty}^{\infty} f(\alpha, \beta) g^{*}(\alpha, \beta) d \alpha d \beta\right| \leq \int_{-\infty}^{\infty} \int_{-\infty}^{\infty}|f(\alpha, \beta)|^{2} d \alpha d \beta
$$

La correlación de dos imágenes, se utiliza en aplicaciones de reconocimiento donde se busca encontrar la mayor correspondencia entre una imagen desconocida y las de una base de datos (patrones) previamente preestablecida. Se calcula la correlación $c(x, y)$ entre esta imagen y todas las de la base de datos, el máximo valor de $c(x, y)$ indica la posición en la que se produce la mayor correspondencia entre la imagen desconocida y la base de datos, figura 7. 


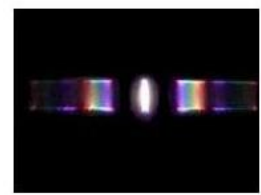

DESCONOCIDO

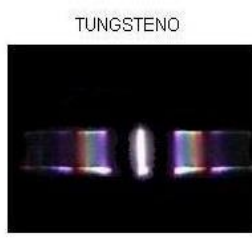

ACERO INOXIDABLE 308L

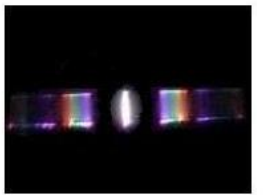

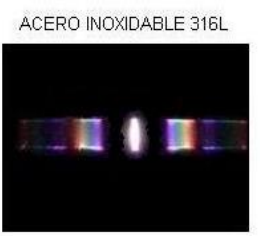

TUNGSTENO CON $2 \%$ TORIO

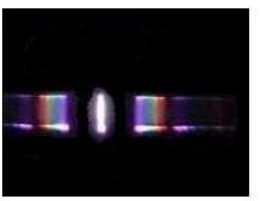

PATRONES

Figura 7: Espectro desconocido y la plantilla de patrones conocidos

\section{Resultados}

En las figuras 8, 9, 10, 11 se muestran los resultados de la correlación cruzada de los espectros de electrodos de Tungsteno puro "desconocido 1", acero inoxidable ER 316L “desconocido 2", acero inoxidable ER 308L "desconocido 3", Tungsteno con 2\% de Thorio “desconocido 4” con la plantilla patrón ver figura 8. Se observó en el gráfico bidimensional y el gráfico tridimensional que la intensidad y el pico del coeficiente de correlación normalizado son diferentes en cada uno de los espectros patrón. Siendo el más intenso y el más largo la posición del espectro patrón que le corresponde al "desconocido".

En las tablas ( 1 y 2 ) se registran los valores de la correspondencia normalizada, de la autocorrelación y la correlación cruzada entre cada uno de los espectros de los electrodos de Tungsteno puro, acero inoxidable ER 316L, acero inoxidable ER 308L, Tungsteno con $2 \%$ de Thorio, aluminio ER 4043 y para acero al carbón ER 70S-6 con cada uno de los patrones. 


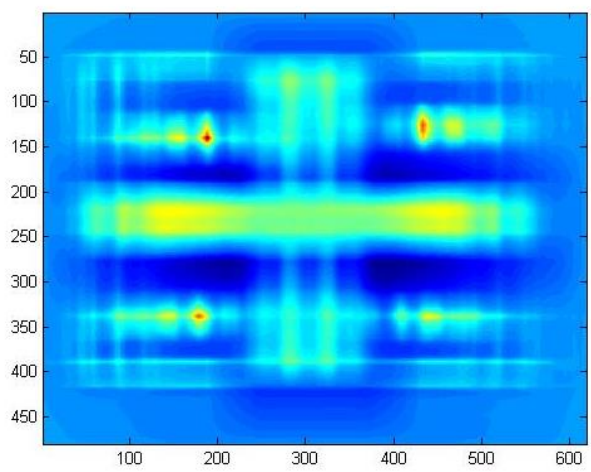

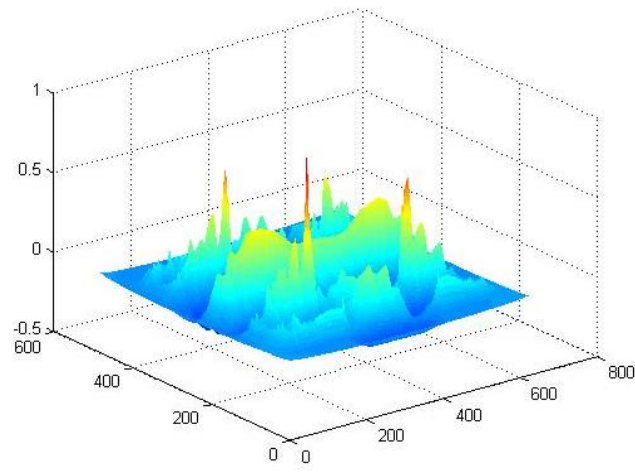

$\mathrm{b}$

Figura 8. a) Ubicación del espectro "desconocido 1" b) pico del coeficiente de correlación normalizado

En la gráfica bidimensional (figura 8 a), se muestra la imagen correspondiente al coeficiente de correlación entre el espectro del electrodo de Tungsteno puro "desconocido 1", y los patrones. Obsérvese la alta intensidad en la posición donde se encuentra el espectro del Tungsteno puro en la plantilla de patrones $(188,141)$. En la gráfica de superficie o tridimensional (figura 8 b), se muestra los picos de la correlación cruzada normalizada. Obsérvese el pico más largo de coeficiente de correlación, en la posición donde se encuentra el espectro del electrodo de Tungsteno puro en la plantilla de patrones.
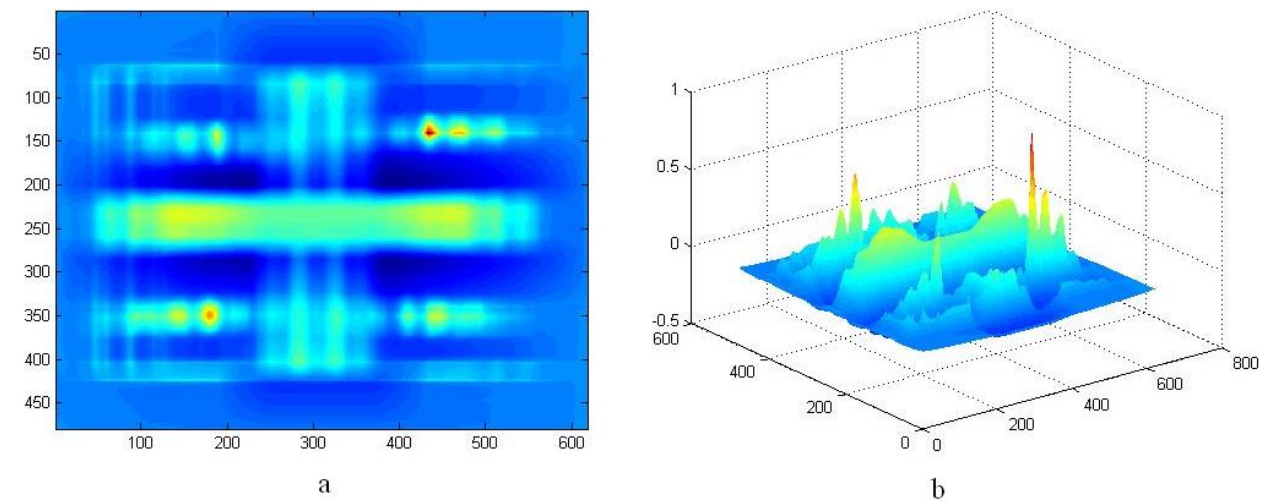

Figura 9. a) Ubicación del espectro "desconocido 2" b) pico del coeficiente de correlación normalizado 
En la gráfica bidimensional (figura 9 a), se muestra la imagen correspondiente al coeficiente de correlación entre el espectro del electrodo de acero inoxidable ER 316L "desconocido 2", y los patrones. Obsérvese la alta intensidad en la posición donde se encuentra el espectro del electrodo de acero inoxidable ER 316L en la plantilla de patrones $(434,141)$.

En la gráfica de superficie o tridimensional (figura 9 b), se muestra los picos de la correlación cruzada normalizada. Obsérvese el pico más largo de coeficiente de correlación, en la posición donde se encuentra el espectro del electrodo de acero inoxidable ER 316L en la plantilla de patrones.
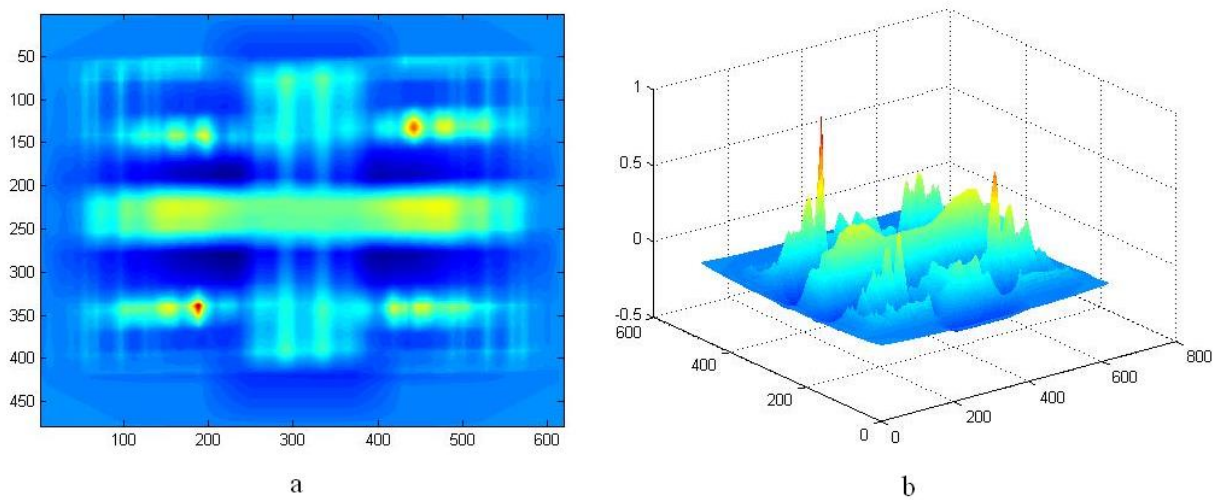

Figura 10. a) Ubicación del espectro "desconocido 3" b) pico del coeficiente de correlación normalizado

En la gráfica bidimensional (figura $10 \mathrm{a}$ ), se muestra la imagen correspondiente al coeficiente de correlación entre el espectro del electrodo de acero inoxidable ER 308L “desconocido 3" y los patrones. Obsérvese la alta intensidad en la posición donde se encuentra el espectro del electrodo de acero inoxidable ER 308L en la plantilla de patrones $(188,340)$.

En la gráfica de superficie o tridimensional (figura 10 b), se muestra los picos de la correlación cruzada normalizada. Obsérvese el pico más largo de coeficiente de correlación, en la posición donde se encuentra el espectro del electrodo ER 308L en la plantilla de patrones. 


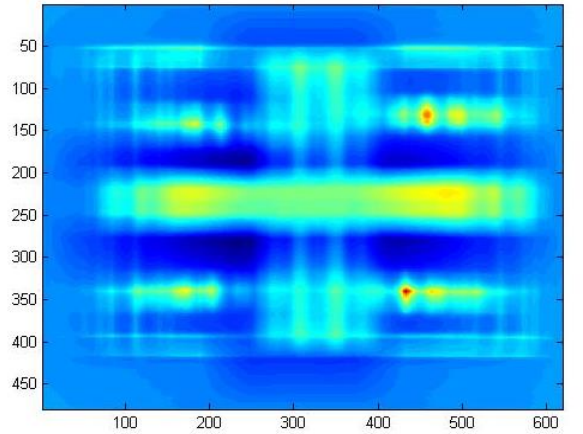

a

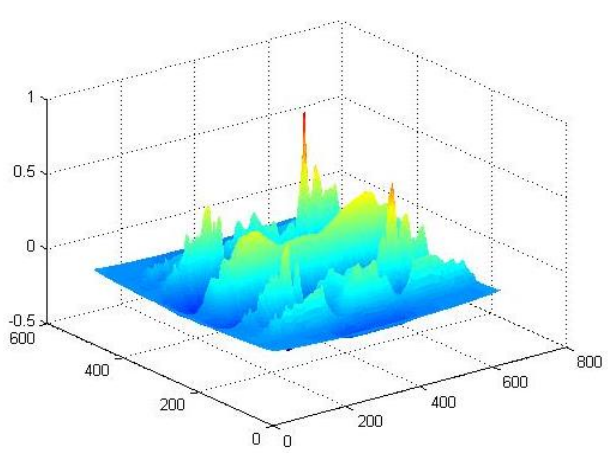

\section{Figura 11. a) Ubicación del espectro "desconocido 4" b) pico del coeficiente de correlación} normalizado

En la gráfica bidimensional (figura 11 a), se muestra la imagen correspondiente al coeficiente de correlación entre el espectro del electrodo de Tungsteno con $2 \%$ de Thorio "desconocido 4" y los patrones. Obsérvese la alta intensidad en la posición donde se encuentra el espectro del electrodo de Tungsteno con $2 \%$ de Thorio en la plantilla de patrones $(434,340)$.

En la gráfica de superficie o tridimensional (figura 11 b), se muestra los picos de la correlación cruzada normalizada. Obsérvese el pico más largo de coeficiente de correlación, en la posición donde se encuentra el espectro del electrodo de Tungsteno con 2\% de Thorio en la plantilla de patrones.

\section{Tabla1}

Resultados de la autocorrelación

\begin{tabular}{|c|c|c|c|}
\hline Electrodos & $\begin{array}{c}\text { Espectros } \\
\text { Imagen en el } \\
\text { dominio espacial }\end{array}$ & $\begin{array}{c}\text { Espectros } \\
\text { Imagen en el dominio } \\
\text { frecuencial }\end{array}$ & $\begin{array}{l}\text { Valor del coeficiente } \\
\text { de autocorrelación } \\
\text { normalizado }\end{array}$ \\
\hline Tungsteno puro & & & 0.9999 \\
\hline $\begin{array}{l}\text { Tungsteno } \\
2 \% \text { Torio }\end{array}$ & & & 1.0000 \\
\hline ER 316L & 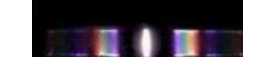 & & 1.0000 \\
\hline
\end{tabular}




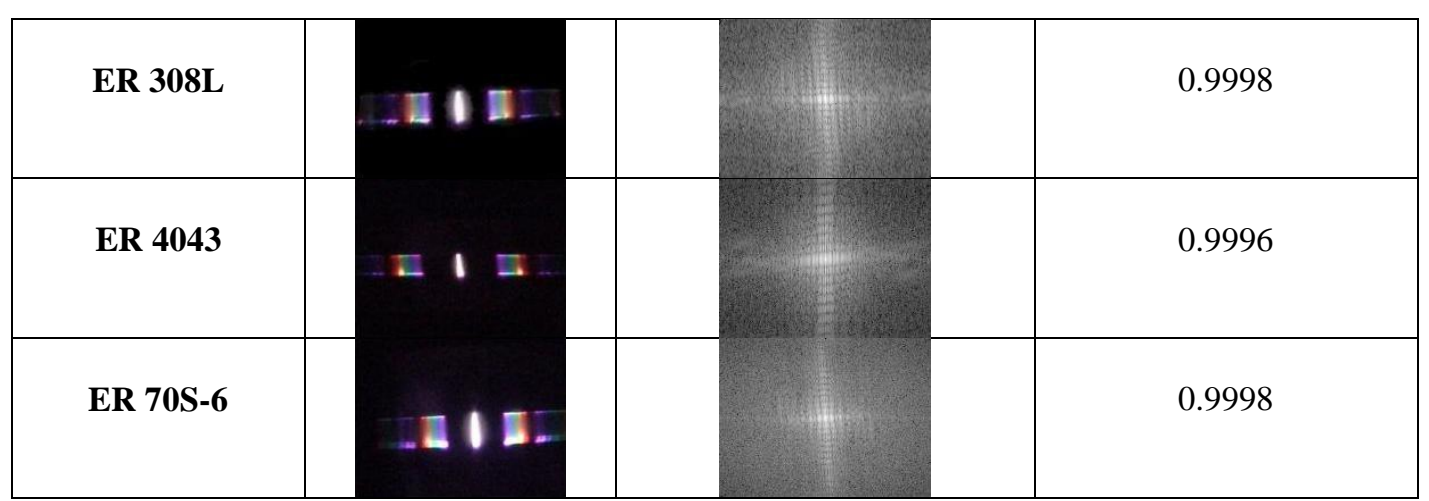

Tabla2

Resultados del coeficiente de correlación normalizado en la correlación de los espectros "desconocidos" y la plantilla de patrones

\begin{tabular}{|l|l|l|l|l|}
\hline "Desconocidos" & $\begin{array}{l}\text { Tungsteno } \\
\text { puro }\end{array}$ & ER 316L & ER 308L & $\begin{array}{l}\text { Tungsteno } \\
\text { 2\% Torio }\end{array}$ \\
\hline Tungsteno puro & $\mathbf{0 . 9 9 9 6}$ & 0.8002 & 0.8005 & 0.8520 \\
\hline ER 316L & 0.8002 & $\mathbf{0 . 9 9 8 9}$ & 0.8102 & 0.7992 \\
\hline ER 308L & 0.8005 & 0.8102 & $\mathbf{0 . 9 9 8 2}$ & 0.7805 \\
\hline Tungsteno 2\% Torio & 0.8520 & 0.7992 & 0.7805 & $\mathbf{0 . 9 9 8 5}$ \\
\hline ER 4043 & 0.6432 & 0.6232 & 0.6406 & 0.5324 \\
\hline ER 70S-6 & 0.7962 & 0.7892 & 0.7988 & 0.7970 \\
\hline
\end{tabular}

En la tabla 1 se muestra los espectros en el dominio del espacio y en el dominio de la frecuencia de cada uno de los electrodos menciona anterior mente y el valor del coeficiente de autocorrelación normalizado y en la tabla 2 se muestra los valores del coeficiente de correlación normalizado de los espectros "desconocido", con cada patrón. Obsérvese que el valor más alto aproximadamente a 1 lo tiene el espectro “desconocido" correspondiente al espectro patrón del mismo electrodo.

De esta manera se recomienda trabajar la Transformada de Fourier y su aplicación en la modelación en el aula, para así, profundizar en el conocimiento del hecho educativo y generar conocimiento científico sobre los procesos de aprendizaje, además se recomienda este tipo de 
problema que sea abordados por los profesores y los libros de textos para los estudiantes y así mantener actualizados los contenidos objeto de aprendizaje en esta disciplina.

\section{Conclusiones}

Los puntos a destacar en esta sección son los siguientes: eficacia de la transformada de Fourier y su inversa para la correlación y el filtrado de imágenes de descargas eléctricas en electrodos industriales. La modelación matemática como un proceso fundamental en la actividad de enseñanza y aprendizaje en el ámbito de la educación científica para afrontar problemas del contexto real interdisciplinario, es decir, contribuyendo en el estudiante; desarrollar habilidad de identificar preguntas selectas, variables, relaciones en la situación del mundo real, trasladar éstas realidad a las matemáticas e interpretar. Se hace énfasis en el hecho didáctico de que la Transformada de Fourier no es una teoría aislada sino de vital importancia y aplicabilidad en el campo científico - tecnológico y en el caso específico del procesado de imágenes de descarga eléctrica.

\section{Referencias}

Alro, H. y Skovsmose, O. (2004). Dialogue and learning in mathematics education. Intention, reflection, critique. Edición Kindle.

Beyer, W. (1994). El discurso y el lenguaje matemáticos en el contexto del aula. Trabajo de grado de maestría no publicado. Universidad Pedagógica Experimental Libertador. Instituto Pedagógico de Caracas, Caracas.

Fernández. D. M., Mejía. K. P., Peña. J.M., Torres (2003). Diseño y desarrollo de un sofware para la discriminación e Identificación de Macérales en muestras de Carbón empleando procesamiento Digital de Imagen y correlación digital. Rev. Col. 35(2).

Gonzalez, R.C., y Wintz P. (1997). Digital Image Processing, Ed. Addison-Wesley. http://sdeuoc.ac.in/sites/default/files/sde_videos/Digital\%20Image\%20Processing\%203rd \%20ed.\%20-\%20R.\%20Gonzalez\%2C\%20R.\%20Woods-ilovepdf-compressed.pdf 
Goodman, J.W. (1968), Introduction to Fourier optics, McGraw-Hill.

Gualdrón, O. (2000). Notas de clase. Universidad Industrial de Santander

Mejía. J. (2004). Apuntes de "Procesamiento Digital de Imágenes". Universidad Autónoma De San Luis Potosí. https://docplayer.es/11449871-Jose-ramon-mejia-vilet-area-decomputacion-e-informatica.html

Mereles, L. (2012). Preprocesamiento de imágenes digitales a través de su Transformada de Fourier. http://lcr.uns.edu.ar/fvc/NotasDeAplicacion/FVC-Leonardo\%20Mereles.pdf

Ohta, Y., Kanade, T., Sakai., T. (1980). Color Information for Region Segmentation, publicado en Computer Graphics and Image Processing. Journal Article, Computer Graphics and Image Processing, 13(3), 222-241. https://www.ri.cmu.edu/pub_files/pub4/ohta_y_1980_1/ohta_y_1980_1.pdf

Pimm, D. (1999). El lenguaje matemático en el aula. Morata.

Serrano, W. (2005). La alfabetización matemática. En: D. Mora (Coord.), R. Becerra, C. Rossetti, W. Serrano, W. Beyer, L. Millán, G. Vernaez, Y. Serres, E. Reverand y A. Rojas, Didáctica crítica, educación crítica de las matemáticas y etnomatemática. Perspectivas para la transformación de la educación matemática en América Latina (pp. 243-276). BoliviaVenezuela: GIDEM- Campo Iris. 